\title{
Indirect coupling of magnons by cavity photons
}

\author{
Babak Zare Rameshti ${ }^{1}$ and Gerrit E. W. Bauer ${ }^{2,3}$ \\ ${ }^{1}$ School of Physics, Institute for Research in Fundamental Sciences (IPM), Tehran 19395-5531, Iran \\ ${ }^{2}$ Kavli Institute of NanoScience, Delft University of Technology, Lorentzweg 1, 2628 CJ Delft, The Netherlands \\ ${ }^{3}$ Institute for Materials Research and WPI-AIMR, Tohoku University, Sendai 980-857r, Japan
}

\begin{abstract}
The interaction between two magnetic spheres in microwave cavities is studied by Mie scattering theory beyond the magnetostatic and rotating wave approximations. We demonstrate that two spatially separated dielectric and magnetic spheres can be strongly coupled over a long distance by standing cavity modes. The interactions splits acoustical (dark) and optical (bright) modes in a way that can be mapped on a molecular orbital theory of the hydrogen molecule. Breaking the symmetry by assigning different radii to the two spheres introduces "ionic" character to the magnonic bonds. These results illustrate the coherent and controlled energy exchange between objects in microwave cavities.
\end{abstract}

\section{INTRODUCTION}

Light-matter systems in which the coherent coupling frequencies exceed the dissipative loss rates are promising elements for solid state quantum information circuits [13]. Spin ensembles may couple strongly to electromagnetic modes of a microwave resonator resulting in hybridized states referred to as magnon polaritons $[6-8,11]$ with the benefit of long coherence [4] and short manipulation [5] times. Here a "magnon" refers to the collective excitation or spin wave of the polarized spin system. Ferro/ferrimagnets can combine a high spontaneous spin density with low damping leading to large cooperativities and narrow linewidths $[12,13]$. The strong, and even ultra-strong coupling regime in which the coupling strength $g$ is comparable to the mode frequencies [14] can therefore be accessed with relative ease. Furthermore, due to the possibility of coupling magnon modes to photons at optical frequencies $[15,16]$, magnetic systems are candidates for coherent conversion of solid state qubits into "flying ones" [17, 18].

On the other hand, controlled creation and read-out of spin-entangled states in quantum information processing with solid state systems remains a major challenge. Coherent coupling of spins can be mediated by a variety of physical mechanisms, such as the magnetic dipolar, exchange, or spin-orbit interaction. The coupling of spins/pseudospins does not have to be direct, but can be realized via an intermediary. This can be localized electrons in a filled shell ion that generate superexchange or the itinerant carriers of metals in the RKKY interaction [19-21]. The non-local exchange coupling can have either sign; it causes the staggered magnetization in magnetic multilayers that display the giant magnetoresistance [22-24]. Quantum systems can also be coupled radiatively over large distances, i.e. when the interaction is mediated by virtual photons in a low-loss resonator or cavity $[25,26]$.

Here we address the hybridization of two magnets by cavity photons. Yttrium iron garnet (YIG), a ferrimagnetic insulator that serves in magnetically tunable filters and resonators at microwave frequencies, can provide high coupling strengths and low damping. YIG's spin density is $2 \cdot 10^{22} \mathrm{~cm}^{-3}$ [27], while its Gilbert constant of the magnetization dynamics typically ranges from $10^{-3}$ to $10^{-5}$ [28-30]. Strong coupling between magnons and cavity photons are manifest in a series of anticrossings in YIG films in coplanar resonators [31-33] and YIG spheres in 3D microwave cavities [12, 13,34].

Soykal et al. [35] reported a quantum theory of photonmagnon coupling in YIG spheres, but this regime has not yet been reached in experiments. Cao et al. modelled the classical magnon-photon coupling for a thin YIG film in a planar cavity and found strong coupling even for spin waves beyond the Kittel mode in microwave transmission and inverse spin Hall effect [8], which was confirmed by experiments $[9,10]$. Our study of the coherent coupling between a YIG sphere and microwave cavity modes [11] revealed that YIG spheres are efficient antennas for microwaves such that (ultra)strong-coupling regimes can be achieved in stand-alone magnetic spheres, as exploited recently [36]. The long-range strong coupling of magnons in spatially separated YIG spheres as mediated by a microwave cavity has been reported [17, 37]. Electrical readout of two distant YIG $\mid \mathrm{Pt}$ bilayers coupled by a microwave cavity mode has been demonstrated recently [38].

Here we extend the classical model [11] to investigate the long-range coupling of magnons in two spatially separated YIG spheres mediated by a microwave cavity, producing a delocalized magnon-polariton hybridized state. The conventional magnetostatic approximation [39, 40], in which the spins interact by the magnetic dipolar field, disregarding exchange as well as propagation effects, is valid in the Rayleigh regime $\lambda \gg a$, where $a$ is the radius of the sphere and $\lambda$ the wavelength of the incident radiation, but breaks down when $\lambda<a$, which is the regime encountered in sub-mm YIG spheres and nanostructured thin films. We therefore study here the properties of the hybridized magnon-polaritons, including retardation effects of microwaves, but disregard the exchange interaction, which is valid for ferromagnets as long as the exchange length $l_{\text {ex }}=\sqrt{2 A /\left(\mu_{0} M_{s}^{2}\right)} \ll a$, with $A$ and $M_{s}$ being the exchange constant and saturation magnetization, respectively. Our results help to picture photon- 
mediated coupling between two or more magnetic samples in terms of the concept of a chemical bond.

This manuscript is organized as follows. In Sec. II, we introduce the details of our model and derive the scattered intensity and efficiency factors for a strongly coupled system of two magnetic spheres in a spherical microwave cavity. In Sec. III, we present and discuss our results that demonstrate the effects both due to the dielectric as well as magnetic effects on the scattering properties and compare our results with experiments. In Sec. IV, we conclude and summarize our findings.

\section{MODEL AND FORMALISM}

Mie expressed a general scattering problem in terms of a rapidly converging expansion into spherical multipole partial waves $[41,42]$. Here we model the indirect coupling of the collective excitations of two magnetic spheres mediated by photons in a spherical cavity by a Mie-like expansion of the coupled Landau-Lifshitz-Gilbert and Maxwell equations. We consider a plane electromagnetic wave with arbitrary polarization and wave vector shining on a cavity loaded by two magnetic spheres with gyromagnetic permeability tensors $\overleftrightarrow{\mu}_{1}$ and $\overleftrightarrow{\mu}_{2}$. A thin spherical shell of a material with high dielectric constant $\epsilon_{c} / \epsilon_{0} \gg 1$, radius $R$, and thickness $\delta$, models a generic resonant cavity. We mimic realistic situations by adjusting the parameters $R$ and $\delta$ (see Fig. 1) to tune the frequencies and broadenings of the cavity modes.

The dynamics of the magnetization vector $\mathbf{M}$ is described by the LLG equation,

$$
\partial_{t} \mathbf{M}=-\gamma \mathbf{M} \times \mathbf{H}_{\mathrm{eff}}+\frac{\alpha}{M_{s}} \mathbf{M} \times \partial_{t} \mathbf{M}
$$

with $\alpha$ and $M_{s}$ being the damping parameter and saturated magnetization, respectively. Effective field $\mathbf{H}_{\mathrm{eff}}=$ $\mathbf{H}_{\text {ext }}+\mathbf{h}$ comprises the external and (collinear) easy axis anisotropy fields $\mathbf{H}_{\text {ext }}$ as well as a distributed ac field $\mathbf{h}(\mathbf{r}, t)$. We linearize the LLG equation by considering the magnetization and driving field vectors

$$
\begin{aligned}
\mathbf{M}(\mathbf{r}, t) & =\mathbf{M}_{0}+\mathbf{m}(\mathbf{r}, t) \\
\mathbf{H}(\mathbf{r}, t) & =\mathbf{H}_{0}+\mathbf{h}(\mathbf{r}, t) .
\end{aligned}
$$

To leading order in the small modulations $\mathbf{m}$ and $\mathbf{h}$ :

$$
\partial_{t} \mathbf{m}=-\gamma\left(\mathbf{M}_{0} \times \mathbf{H}_{\mathrm{eff}}^{(1)}+\mathbf{m} \times \mathbf{H}_{\mathrm{eff}}^{(0)}\right)+\frac{\alpha}{M_{s}} \mathbf{M}_{0} \times \partial_{t} \mathbf{m}
$$

where $\mathbf{H}_{\mathrm{eff}}^{(0)}=\mathbf{H}_{\mathrm{ext}}$ and $\mathbf{H}_{\mathrm{eff}}^{(1)}=\mathbf{h}$. In the frequency domain, for $\mathbf{H}_{\text {ext }}$ and $\mathbf{M}_{0} \| \hat{z}$,

$$
i \omega \mathbf{m}=\mathbf{z} \times\left(\omega_{\mathrm{M}} \mathbf{h}-\omega_{\mathrm{H}} \mathbf{m}+i \omega \alpha \mathbf{m}\right)
$$

with $\omega_{\mathrm{M}}=\gamma M_{s}$ and $\omega_{\mathrm{H}}=\gamma H_{0}$. We express Eq. (4) as

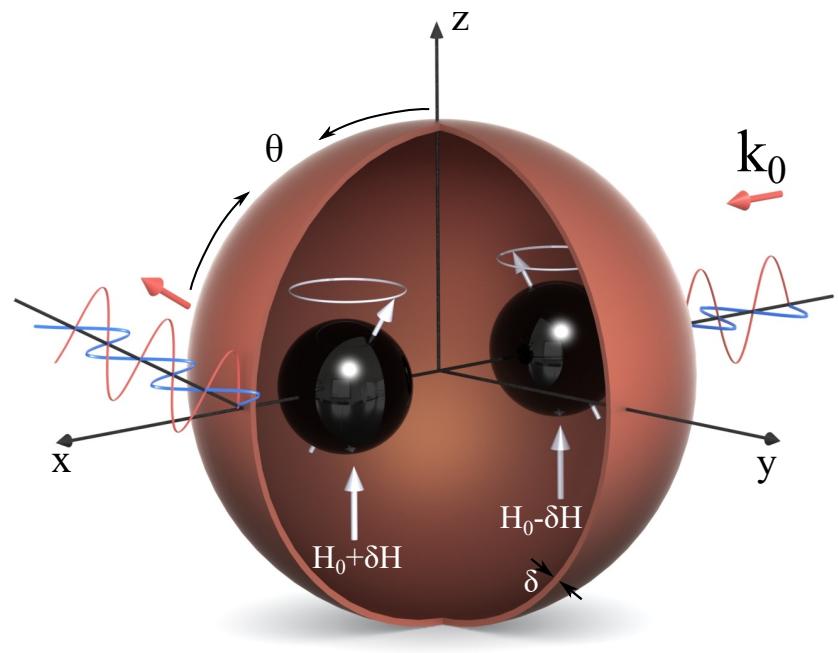

Figure 1. (Color online) A plane electromagnetic wave illuminates a large spherical cavity from an arbitray direction. The latter is modeled by a dielectric spherical shell of radius $R$, thickness $\delta$, and permittivity $\epsilon_{c}$. Two magnetic spheres of radius $a_{1}$ and $a_{2}$ are located at antinodes of the ac magnetic field of the $(2,2)$ and $(2,-2)$ confinement modes of the cavity, i.e., at $\mathbf{d}_{1}$ and $\mathbf{d}_{2}$ on the $\mathbf{x}$ axis. A constant magnetic field $H_{0}$ saturates the equilibrium magnetizations. The scattered waves are measured by a detector in the far field as a function of the scattering angles, here $(\theta, \varphi)=(\pi / 2, \pi)$.

$\mathbf{m}=\overleftrightarrow{\chi} \cdot \mathbf{h}$ in terms of the magnetic permeability tensor

$$
\begin{aligned}
\overleftrightarrow{\mu} & =\mu_{0}(\overleftrightarrow{\mathrm{I}}+\overleftrightarrow{\chi}) \\
& =\mu_{0}\left(\begin{array}{ccc}
1+\chi & -i \kappa & 0 \\
i \kappa & 1+\chi & 0 \\
0 & 0 & 1
\end{array}\right)
\end{aligned}
$$

where

$$
\begin{aligned}
\chi & =\frac{\left(\omega_{\mathrm{H}}-i \alpha \omega\right) \omega_{\mathrm{M}}}{\left(\omega_{\mathrm{H}}-i \alpha \omega\right)^{2}-\omega^{2}}, \\
\kappa & =\frac{\omega \omega_{\mathrm{M}}}{\left(\omega_{\mathrm{H}}-i \alpha \omega\right)^{2}-\omega^{2}} .
\end{aligned}
$$

The Maxwell equations inside a homogeneous sphere at frequency $\omega$ read

$$
\begin{aligned}
\nabla \times \mathbf{E} & =i \omega \mathbf{b} ; \quad \nabla \times \mathbf{h}=-i \omega \epsilon_{\mathrm{sp}} \mathbf{E} \\
\nabla \cdot \mathbf{E} & =0 ; \quad \nabla \cdot \mathbf{b}=0 .
\end{aligned}
$$

The magnetic induction $\mathbf{b}$ and the magnetic field $\mathbf{h}$ inside this medium are related by

$$
\mathbf{b}=\overleftrightarrow{\mu} \cdot \mathbf{h}, \quad \mathbf{D}=\epsilon_{\mathrm{sp}} \mathbf{E}
$$

and $\mathbf{b}$ satisfies the wave equation

$$
\nabla \times \nabla \times\left(\mu_{0} \overleftrightarrow{\mu}^{-1} \cdot \mathbf{b}\right)-k_{\mathrm{sp}}^{2} \mathbf{b}=0
$$

where $k_{\mathrm{sp}}^{2}=\omega^{2} \epsilon_{\mathrm{sp}} \mu_{0}$ and $\epsilon_{\mathrm{sp}}$ is the scalar permittivity of the medium. Keeping Eq. (11) in mind, we expand $\mathbf{h}$ in 
terms of vector spherical waves as

$$
\mathbf{h}=\sum_{n m} \bar{\eta}_{n m}\left[d_{m n} \mathbf{V}_{n m}^{(1)}(k, \mathbf{r})+c_{m n} \mathbf{N}_{n m}^{(1)}(k, \mathbf{r})\right]
$$

where $k$ is as yet undetermined, $n$ runs from 1 to $\infty$, and $m=-n, \cdots, n$. The prefactors read $\bar{\eta}_{n m}=\eta_{n m} k_{0} /\left(\omega \mu_{0}\right)$ with

$$
\eta_{n m}=i^{n} E_{0}\left[\frac{2 n+1}{n(n+1)} \frac{(n-m) !}{(n+m) !}\right]^{1 / 2}
$$

where $E_{0}$ is the amplitude of the electric field of the incident wave. The vector spherical wave functions are defined as

$$
\begin{aligned}
\mathbf{V}_{n m}^{(j)}(k, \mathbf{r}) & =z_{n}^{(j)}(k r) \mathbf{X}_{n m}(\mathbf{r}), \\
k \mathbf{N}_{n m}^{(j)}(k, \mathbf{r}) & =\nabla \times \mathbf{V}_{n m}^{(j)}(k, \mathbf{r}) .
\end{aligned}
$$

where $z_{n}^{(j)}$ are spherical Bessel functions of the $j$-th kind, e.g., $z_{n}^{(3)}=h_{n}^{(1)}$ is the spherical Bessel functions of the third kind (Hankel function). $\mathbf{X}_{n m}=$ $\mathbf{L} Y_{n m}(\hat{r}) / \sqrt{n(n+1)}$, where $Y_{n m}(\hat{r})$ are spherical (surface) harmonics and $\mathbf{L}=-i \mathbf{r} \times \nabla_{r}$ is the angular momentum and $\nabla_{r}$ the gradient operator. By invoking the vector spherical wave function expansion for $\mathbf{b}$ and $\overleftrightarrow{\mu}^{-1} \cdot \mathbf{b}$ in the wave equation Eq. (13) leads to the dispersion relation for $k(\omega)$. We focus in the following on the lowest frequency resonances for a given angular momentum without radial nodes in the sphere. For simplicity of notation we therefore omit the "main quantum number" when labelling the cavity modes.

The electric field distribution is obtained by $\mathbf{E}=$ $(i / \omega c) \nabla \times \mathbf{h}$. We expand the incident fields $\mathbf{E}_{\text {inc }}, \mathbf{h}_{\text {inc }}$ and scattered fields $\mathbf{E}_{s}, \mathbf{h}_{s}$ outside the sphere analogously. The scattered field reads then

$$
\mathbf{h}_{s}=\sum_{n m} \bar{\eta}_{n m}\left[b_{m n} \mathbf{N}_{n m}^{(3)}+a_{m n} \mathbf{V}_{n m}^{(3)}\right]
$$

with $k_{0}^{2}=\omega^{2} \epsilon_{0} \mu_{0}$. The expansion coefficients $a_{n m}$ and $b_{n m}$ are determined by the boundary conditions. We consider the situation that the magnetic sphere is illuminated by a plane wave with arbitrary polarization and direction of incidence as indicated in Fig. (1). This incident fields can be expanded as,

$$
\mathbf{h}_{\mathrm{inc}}=-\sum_{n m} \bar{\eta}_{n m}\left[q_{m n} \mathbf{N}_{n m}^{(1)}+p_{m n} \mathbf{V}_{n m}^{(1)}\right]
$$

with coefficients

$$
\begin{aligned}
& p_{m n}=\frac{\eta_{n m}}{i^{n} E_{0}}\left[p_{\theta} \tau_{m n}\left(\cos \theta_{k}\right)-i p_{\phi} \pi_{m n}\left(\cos \theta_{k}\right)\right] e^{-i m \phi_{k}} \\
& q_{m n}=\frac{\eta_{n m}}{i^{n} E_{0}}\left[p_{\theta} \pi_{m n}\left(\cos \theta_{k}\right)-i p_{\phi} \tau_{m n}\left(\cos \theta_{k}\right)\right] e^{-i m \phi_{k}}
\end{aligned}
$$

where $\hat{\mathbf{p}}=\left(p_{\theta} \hat{\boldsymbol{\theta}}_{k}+p_{\phi} \hat{\boldsymbol{\phi}}_{k}\right)$ is the normalized complex polarization vector, with unit vectors $\hat{\boldsymbol{\theta}}_{k}$ and $\hat{\boldsymbol{\phi}}_{k},|\hat{\mathbf{p}}|=1$ and $\theta_{k}\left(\phi_{k}\right)$ is the polar (azimuthal) angle of incidence. Two auxiliary functions are defined by

$$
\pi_{m n}(\cos \theta)=\frac{m}{\sin \theta} P_{n}^{m}(\cos \theta), \tau_{m n}(\cos \theta)=\frac{d}{d \theta} P_{n}^{m}(\cos \theta)
$$

All fields of the scattering problem are now expanded in terms of vector spherical wave functions. The boundary conditions

$$
\begin{aligned}
{\left[\mathbf{E}_{\text {inc }}+\mathbf{E}_{s}\right] \times \mathbf{e}_{\mathbf{r}} } & =\mathbf{E}_{i} \times \mathbf{e}_{\mathbf{r}}, \\
{\left[\mathbf{h}_{\text {inc }}+\mathbf{h}_{s}\right] \times \mathbf{e}_{\mathbf{r}} } & =\mathbf{h}_{i} \times \mathbf{e}_{\mathbf{r}}
\end{aligned}
$$

can be rewritten in terms of the transmission matrix $\mathcal{T}$ that relates the scattered to the incoming fields

$$
\left(\begin{array}{l}
a_{n m} \\
b_{n m}
\end{array}\right)=\mathcal{T}\left(\begin{array}{c}
p_{n m} \\
q_{n m}
\end{array}\right) .
$$

We are interested in more than one scattering objects in the cavity. In order to describe the collective excitations of non-overlapping magnetic spheres, we expand the total incident field striking the surface of the $i$-th sphere, the initial incident waves, and the scattered field of the other spheres with index $j \neq i$, in the coordinate systems centered at sphere $i$ as

$$
\mathbf{E}_{\mathrm{inc}}^{i}=\mathbf{E}_{\mathrm{inc}}+\sum_{j \neq i} \mathbf{E}_{s}^{j} ; \quad \mathbf{h}_{\mathrm{inc}}^{i}=\mathbf{h}_{\mathrm{inc}}+\sum_{j \neq i} \mathbf{h}_{s}^{j}
$$

The transformation of waves scattered by one sphere into incident waves for the other one is formulated by the addition theorem of vector spherical harmonics [43], i.e., the expansion of the basis set in a translated reference system. By transforming the wave scattered by one sphere to a coordinate system centered at the other and imposing appropriate boundary conditions, we arrive at the scattering coefficients

$$
\left(\begin{array}{c}
a_{n m}^{i} \\
b_{n m}^{i}
\end{array}\right)=\mathcal{T}^{i}\left[\left(\begin{array}{c}
p_{n m}^{i} \\
q_{n m}^{i}
\end{array}\right)+\sum_{j \neq i} \mathcal{R}^{j i}\left(\begin{array}{c}
a_{n m}^{j} \\
b_{n m}^{j}
\end{array}\right)\right],
$$

where the superscript indicates the coordinate system centered at sphere $i$ and $\mathcal{R}^{j i}$ is the translation matrix from sphere $j$ to $i$ [43]. The second term on the righthand side represents the multiple scattering between the objects. The scattering coefficients in the coordinate system of the cavity can be obtained by the unitary transformation $\mathcal{R}^{i 0}$ defined by the addition theorem

$$
\left(\begin{array}{c}
a_{n m}^{0} \\
b_{n m}^{0}
\end{array}\right)=\mathcal{R}^{i 0}\left(\begin{array}{c}
a_{n m}^{i} \\
b_{n m}^{i}
\end{array}\right)
$$

These expressions are sufficient to compute the scattering matrix for the entire system.

In order to make contact with experiments, we consider the far-field limit, in which the intensity of the two polarization components $I_{\theta}$ and $I_{\phi}$ are

$$
I_{\theta} \sim \frac{E_{0}^{2}}{k_{0}^{2} r^{2}}\left|S_{1}(\theta, \phi)\right|^{2}, \quad I_{\phi} \sim \frac{E_{0}^{2}}{k_{0}^{2} r^{2}}\left|S_{2}(\theta, \phi)\right|^{2}
$$


where $\theta(\phi)$ is the polar (azimuthal) angle of the observer at distance $r$ and scattering intensity functions are

$$
\begin{aligned}
& S_{1}(\theta, \phi)=\sum_{n m}\left[a_{m n} \tilde{\tau}_{m n}(\cos \theta)+b_{m n} \tilde{\pi}_{m n}(\cos \theta)\right] e^{i m \phi}, \\
& S_{2}(\theta, \phi)=\sum_{n m}\left[a_{m n} \tilde{\pi}_{m n}(\cos \theta)+b_{m n} \tilde{\tau}_{m n}(\cos \theta)\right] e^{i m \phi},
\end{aligned}
$$

We define a dimensionless scattering efficiency factor $Q_{\text {sca }}$ as the total (i.e. angular integrated) scattering cross section of the light intensity divided by the geometrical area $\pi R^{2}$ as,

$$
Q_{\text {sca }}=\frac{4}{k_{0}^{2} R^{2}} \sum_{n m}\left(\left|a_{n m}\right|^{2}+\left|b_{n m}\right|^{2}\right)
$$

The efficiency factor $Q_{\text {ext }}$ defined analogously for the total extinction cross section

$$
Q_{\text {ext }}=\frac{4}{k_{0}^{2} R^{2}} \sum_{n m} \operatorname{Re}\left(p_{n m}^{*} a_{n m}+q_{n m}^{*} b_{n m}\right)
$$

measures the total energy loss of the incident beam by absorption and scattering.

$$
Q_{\mathrm{abs}}=Q_{\mathrm{ext}}-Q_{\mathrm{sca}}
$$

reflects the loss of intensity due to Gilbert damping in the sample.

\section{RESULTS}

The observables defined above can be computed numerically as a function of material and cavity parameters. We focus here on a spherical cavity with fixed radius $(R=4 \mathrm{~mm})$ loaded with two dielectric spheres at a fixed distance $d_{0}=2.5 \mathrm{~mm}$, but with adjustable diameter, as in Fig. 1. We focus on the strong coupling regime in which the polaritonic mode splitting is comparable or larger than the dissipation, i.e. we have spectrally sharp cavity modes and not too large Gilbert damping. Without using the macrospin approximation, we focus our discussion to the nearly uniform (Kittel) mode that displays the strongest coupling to the microwaves [8].

Forward scattered intensities, i.e., $\theta=\pi / 2, \phi=\pi$, and scattering efficiency factors are convenient and observable measures of the microwave-matter coupling. In order to compare our results with recent experiments, we adopt parameters for YIG with gyromagnetic ratio $\gamma /(2 \pi)=28$ $\mathrm{GHz} / \mathrm{T}$, saturation magnetization $\mu_{0} M_{s}=175 \mathrm{mT}$ [44], Gilbert damping constant $\alpha=3 \times 10^{-4}$ [28-30], and relative permittivity $\epsilon_{\mathrm{sp}} / \epsilon_{0}=15$ [45]. The incident microwave radiation comes from the positive $\mathbf{x}$ direction $\left(\theta_{k}=\pi / 2\right.$ and $\left.\phi_{k}=0\right)$ and is linearly polarized such that its electric/magnetic components are in the $-\mathbf{z} / \mathbf{y}$ directions (static magnetic field and magnetization $\mathbf{H}_{0} \| \mathbf{z}$ ).
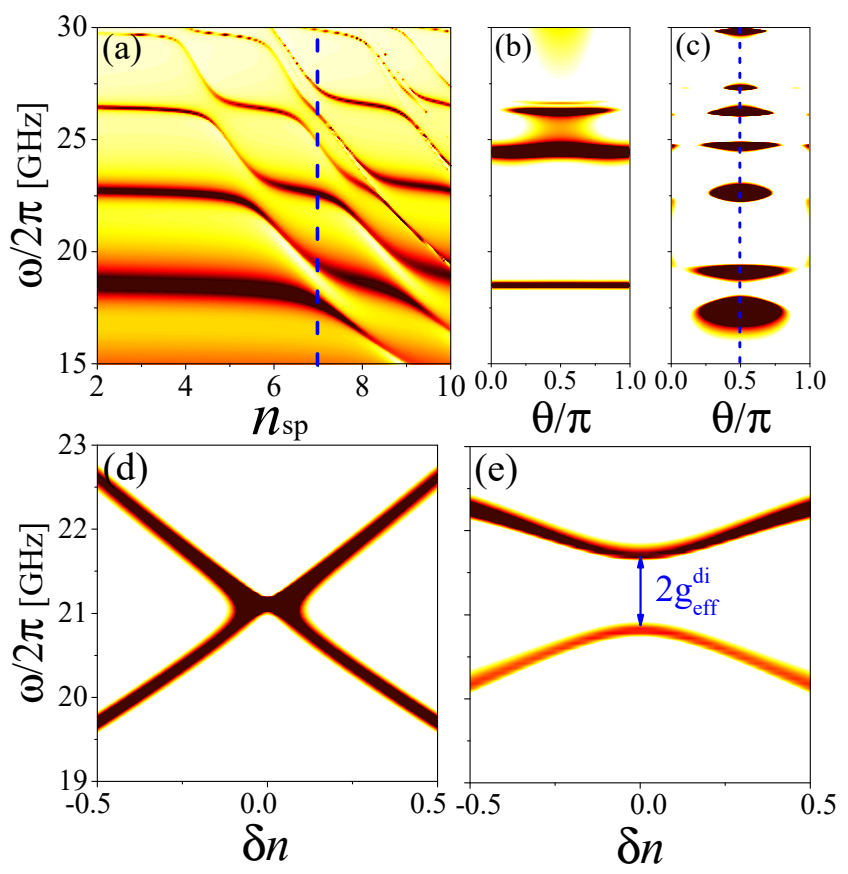

Figure 2. (Color online) (a): The scattering efficiency factor Eq. (33) for two non-magnetic dielectric spheres of radius $a_{1}=a_{2}=1 \mathrm{~mm}$, cavity radius $R=4 \mathrm{~mm}$, and asymmetry $\delta n=1$ plotted as a function of frequency $\omega / 2 \pi$ and average refractive index $n_{\mathrm{sp}}$. (b) and (c): The scattering intensity $\left|S_{1}\right|^{2}$ as function of scattering angle $\theta$ and frequency $\omega / 2 \pi$ plotted for the same spheres $\left(n_{\mathrm{sp}}=7, \delta n=1\right)$ without and with cavity, respectively, while (d) and (e) are the corresponding scattering efficiencies. The anticrossing in (e) reveals the interaction with the cavity field by the coupling strength $2 g_{\mathrm{eff}}^{\mathrm{di}}$, i.e., the frequency splitting of the modes at $\delta n=0$. The dashed lines are guides for the eye.

We also investigate the dependence of the observables on the scattering angle with respect to the outgoing radiation.

We start by studying the effects of asymmetry on the photon-mediated coupling of two non-magnetic spheres with refractive indices $n_{1}=n_{\mathrm{sp}}+\delta n$ and $n_{2}=n_{\mathrm{sp}}-\delta n$. In Fig. 2(a) the scattering efficiency factor Eq. (33) is plotted as a function of frequency $\omega /(2 \pi)$ and average refractive index $n_{\mathrm{sp}}=\sqrt{\epsilon_{\mathrm{sp}} / \epsilon_{0}}$ of the spheres with $a=1$ $\mathrm{mm}$ in a spherical cavity with radius $R=4 \mathrm{~mm}$ and broken symmetry with $\delta n=1$. The spheres are placed at the local maxima of the electric field distribution of the cavity, i.e., $\mathbf{d}_{1}=d_{0} \mathbf{x}$ and $\mathbf{d}_{2}=-d_{0} \mathbf{x}$, respectively, where $d_{0}=2.5 \mathrm{~mm}$. This ensures a significant coupling strength and nearly uniform distribution of the cavity field over the spheres.

When $\delta n \neq 0$ the individual resonances of the two spheres are distinguishable in Fig. 2(a). Not only the lowest but also higher plasmonic modes $\left(\sim n_{\mathrm{sp}}^{2}\right)$ anticross strongly with the (constant) cavity resonances. The angular dependence of the scattering without and with cavity is plotted in panels (b) and (c) of Fig. 2, respectively. The 


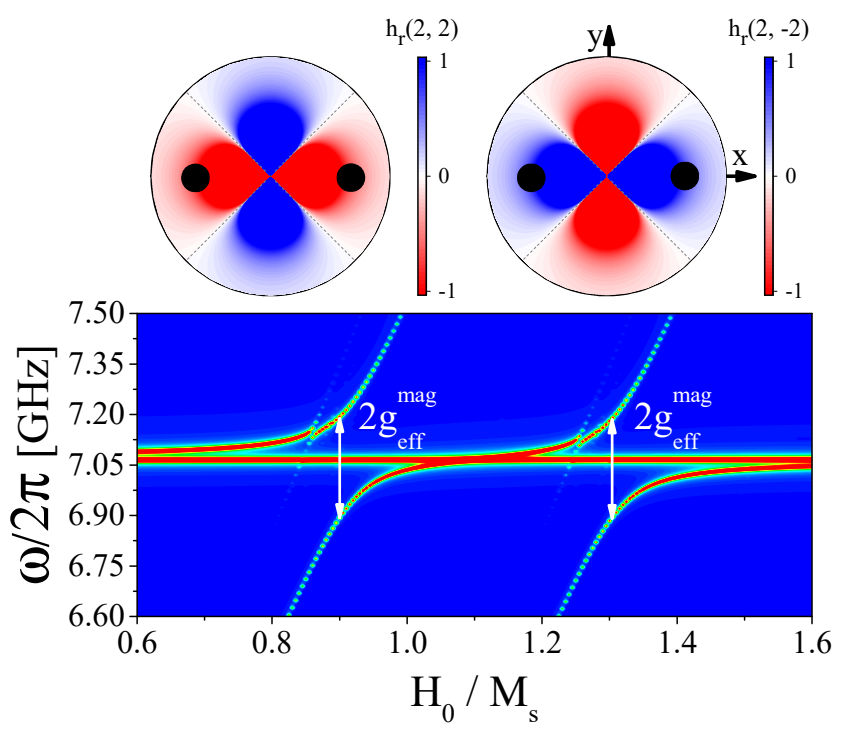

Figure 3. (Color online) Scattering efficiency factor $Q_{\text {sca }}$ as function of magnetic field $H_{0} / M_{s}$ and frequency $\omega / 2 \pi$ for two YIG spheres of radius $a_{1}=a_{2}=0.5 \mathrm{~mm}$ and relative permittivity $\epsilon_{\mathrm{sp}} / \epsilon_{0}=15$ in a spherical cavity of radius $R=4 \mathrm{~mm}$ on the two antinodes of the cavity mode at $\omega_{2} / 2 \pi \sim 7.05 \mathrm{GHz}$ is shown in bottom panel. The field at each sphere is detuned by $|\delta H| / M_{s} \sim 0.2$ with opposite sign. $g_{\text {eff }}^{\mathrm{mag}}$ is the magnon-cavity coupling strength. The radial component of microwave magnetic field $h_{r}$ for the cavity mode frequency $\omega_{2}$ in the equator plane is shown in top panel, the black circles indicate two spheres.

eigenmodes of the two coupled-dielectric spheres have a predominant $s$-wave character when the wavelength $\lambda \gtrsim a \sqrt{\epsilon_{\mathrm{sp}} / \epsilon_{0}}$, i.e. no scattering-angle dependence in the regime in which no resonant states are formed.

The radiative coupling between two dielectric spheres by the cavity eigenmodes is revealed by tuning the resonances with the asymmetry parameter $\delta n \in[-0.5,0.5]$ for $n_{\mathrm{sp}}=7$. Fig. 2(d) and (e) are plots of the scattering efficiency factor $Q_{\text {sca }}$ as a function of frequency $\omega / 2 \pi$ and asymmetry $\delta n$ in the absence and presence of the external cavity, respectively. The photon-mediated coupling corresponds to the splitting at the nominal crossing point $(\delta n=0)$ and found to be $g_{\mathrm{eff}}^{\mathrm{di}} / 2 \pi \sim 0.6 \mathrm{GHz}$, which is much larger that the broadening and therefore "strong". Removing the cavity suppresses the splitting, as seen in Fig. 2(d), proving that the direct dipolar coupling between the spheres and the multiple scattering of the microwaves between spheres in the absence of a cavity are weak. In analogy with plasmonic molecules in metallic nanostructures [46], which are bound by the optical near-fields, we refer to this hybridized state as a plasmonpolariton molecule.

The magnetism of the spheres affects the microwave scattering properties strongly, but the plasmonic effects causing hybridization of the resonances of cavity and sphere remain to be very relevant. Our results help to interpret recent experimental results on cavity-mediated

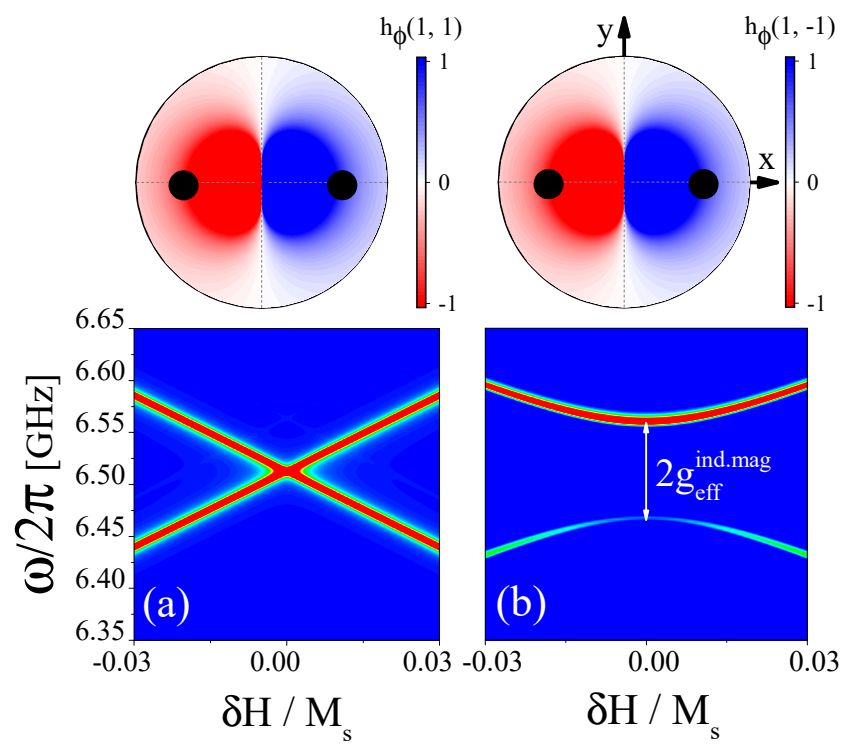

Figure 4. (Color online) (a) and (b): Scattering efficiency factor $Q_{\text {sca }}$ as function of $\omega / 2 \pi$ and $\delta H / M_{s}$ for the same two spheres as Fig. (3) without and with cavity, respectively, but the detuning is much smaller than in Fig. (3). $H_{0} / M_{s}=1$ is fixed such that the magnetostatic modes of each sphere are detuned from $\omega_{1}$. The anticrossing in (b) illustrates the coupling of the two YIG spheres; the non-local magnon-magnon coupling strength $g_{\text {eff }}^{\text {ind.mag }}$ is the frequency splitting of the modes at $\delta H=0$. The azimuthal component of microwave magnetic field $h_{\phi}$ for the cavity mode frequency $\omega_{1}$ in the equator plane is shown in top panel, the black circles indicate two spheres.

coupling of two YIG spheres [37] by taking into acount the finite size of the spheres and cavity-field distribution. Fig. 3 shows the scattering efficiency factor as a function of frequency $\omega / 2 \pi$ and uniform magnetic field $H_{0} / M_{s}$ for our spherical cavity containing now two YIG spheres with radii $a_{1}=a_{2}=0.5 \mathrm{~mm}$. The frequency of the microwaves with wave vector along the $x$-direction is tuned to the 5 -fold degenerate cavity modes with $n=2(d$ wave); $\omega_{2} / 2 \pi \sim 7.05 \mathrm{GHz}$, of which only the $\omega_{2, \pm 2}$ states are excited by symmetry. An asymmetry is now induced by a detuning magnetic field with opposite sign on different spheres $\delta H= \pm 0.2 M_{s}$. The two spheres occupy antinodes of the $p$ and $d$ cavity resonances shown in the top panels of Figs. 3 and 4 with parameters chosen to be close to the experiment [37]. Two distinct anticrossings are the signature of mixed magnon-polariton modes with a magnon-photon coupling of $g_{\mathrm{eff}}^{\mathrm{mag}} / 2 \pi \sim 150 \mathrm{MHz}$ between the Kittel modes of both spheres and the cavity mode. Small satellites indicate the coupling to a higher ("Walker") mode in both spheres. Fig. 3 also shows a cavity mode that is not affected by the magnets [17]. This mode is a linear combination of the active cavity modes $\omega_{2, \pm 2}$ that does not couple to the sphere. Although the spherical symmetry of the empty cavity has been broken by the load, the axial symmetry remains intact and is responsible for this effect.

Next we fix $H_{0} / M_{s}=1$ and study the effect of small de- 


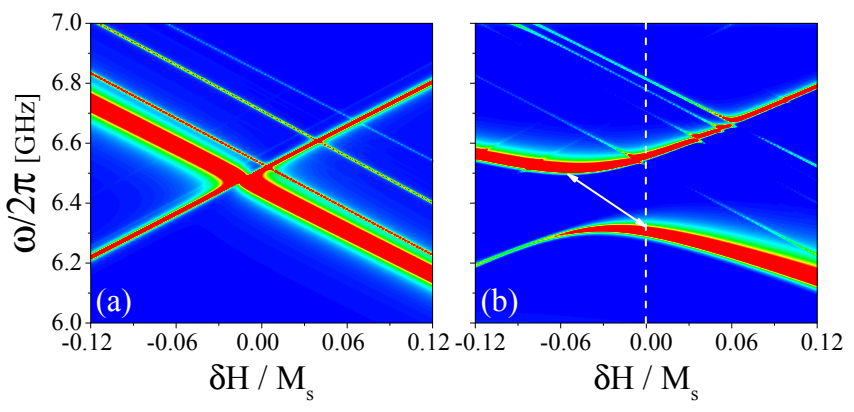

Figure 5. (Color online) Scattering efficiency as function of frequency $\omega / 2 \pi$ and normalized bias field $\delta H / M_{s}$ for two YIG spheres $\left(\epsilon_{\mathrm{sp}} / \epsilon_{0}=15\right)$ with radius $a_{1}=0.5 \mathrm{~mm}$ and $a_{2}=1$ $\mathrm{mm}$ (a) without cavity and (b) in a spherical cavity of radius $R=4 \mathrm{~mm}$. The white arrow in (b) illustrates the "indirect gap" induced by the radiative coupling.

tunings $\delta H / M_{s}$ in the dispersive regime. In Figs. 4(a) and (b) the Kittel mode lies above the $p$-wave cavity eigenmode $\omega_{1} / 2 \pi \sim 6 \mathrm{GHz}$. Note that the scattering efficiencies in the dispersive regime are much smaller than those in Fig. 3. Panel (a) shows results for two YIG spheres of radii $a=0.5 \mathrm{~mm}$ without cavity, while for panel (b) the spherical cavity has been added. The anticrossing in Fig. 4(b) illustrates that the magnons of the two magnets interact over long distances through the virtual exchange of cavity microwave photons. The coupling strength is given by the frequency splitting of the modes at $\delta H=0$, giving a value of $g_{\text {eff }}^{\text {ind.mag }} / 2 \pi \sim 43 \mathrm{MHz}$. This coupling requires an external resonator, cf. Fig. 4(a), and can therefore not be explained by the direct magnetic dipolar interactions or multiple scattering between the spheres, as observed [37].

We observe that the upper mode has a relatively large oscillator strength ("bright mode"), while the lower mode intensity is suppressed at $\delta H=0$ ("dark mode"). The order and symmetry of these modes depends on the sign of the magnon-cavity mode detuning as well as the phase relation between the amplitude of the cavity mode on the spheres. In principle, many modes contribute, but the ones closest in frequency dominate. The higher frequency mode in 4(a) is the "acoustic" (symmetric) mode that strongly interacts with the low frequency mode $\omega_{1}$, which has the largest oscillator strength for forward scattering. The lower "optical" (antisymmetric) mode for $\delta H=0$ interacts with (and is pushed to lower frequencies) by mode $\omega_{2}$. The scattering power of the $\omega_{2}$ mode (without load) is much weaker than that of $\omega_{1}$, which renders the lower collective magnetic mode to be "dark". We note that the "darkness" is not absolute, since the remaining intensity does not vanish for $\delta H=0$ and depends on the details of the system and scattering configuration.

Lambert et al. [37] find that a cavity mode $\omega_{2} / 2 \pi \sim$ 7.15 GHz couples with the Kittel mode of a YIG sphere with $a=0.5 \mathrm{~mm}$ by $g_{2} / 2 \pi\left(\equiv g_{\mathrm{eff}}^{\mathrm{mag}} / 2 \pi\right) \approx 150 \mathrm{MHz}$, in excellent agreement with our calculations. By a dispersive measurement technique they also observe a split-
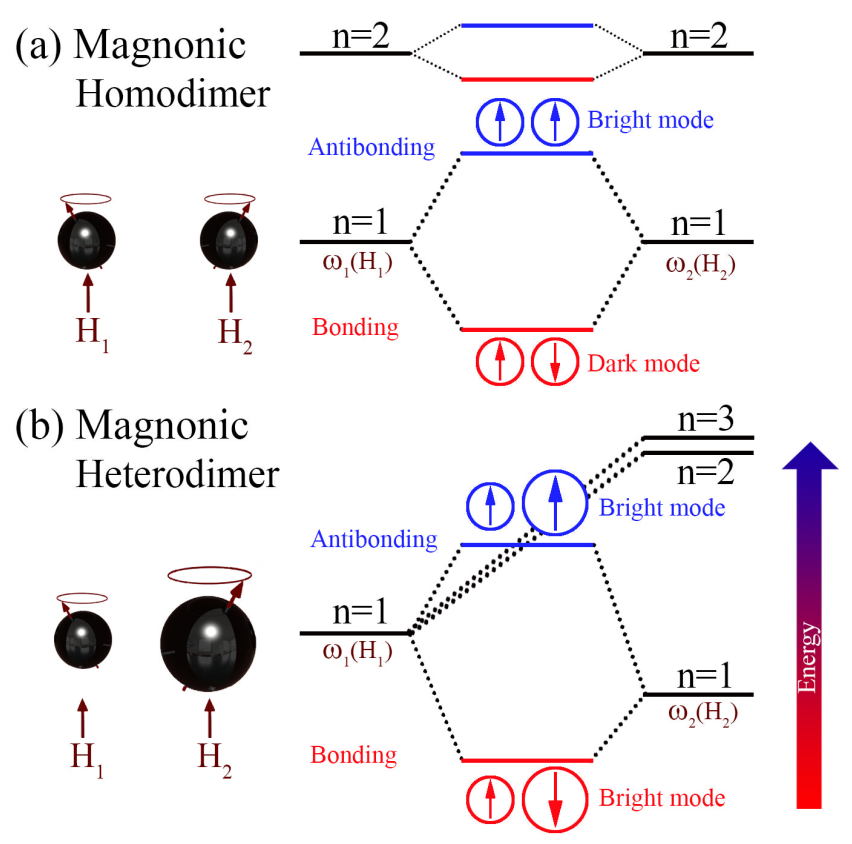

Figure 6. (Color online) Energy level diagram describing the magnon hybridization in analogy with chemical bonds resulting from the interaction between two spheres via microwave cavity modes. (a) Magnonic homodimer consists of two similar magnetic spheres subjected to local magnetic fields $H_{1(2)}=$ $H_{0} \pm \delta H$, and (b) Magnonic heterodimer consists of two dissimilar magnetic spheres. In (a) magnon hybridization only occurs between magnonic states of the same angular momentum denoted by $n_{i}$, while the reduced symmetry in a heterodimer introduces coupling between all modes. In a homodimer, the bonding level is dark since it has no dipole moment, while the antibonding level is bright. In a heterodimer all modes are visible. The arrows in circles indicate the relative magnonic phase (not spin or equilibrium magnetization).

ting which they interpret in terms of in-phase and outof-phase precessions of the individual magnetization dynamics. The observed splitting of these two modes agrees well with the calculated ones, i.e. $2 J / 2 \pi=87 \mathrm{MHz}$ as compared to our $2 g_{\text {eff }}^{\text {indmag }} / 2 \pi \sim 86 \mathrm{MHz}$. The order of "bright" and "dark" modes is opposite to what we find in Fig. 4. This discrepancy is caused by the relative low frequency $\omega_{1} / 2 \pi \sim 3.55 \mathrm{GHz}$ in the experiments, which is not reproduced by our spherical cavity in which $\omega_{1} / 2 \pi \sim 6 \mathrm{GHz}$.

For two identical spheres the scattering properties $\mathcal{A}$, such as $Q_{\text {sca }}$, are parity (mirror) symmetric in parameter space, i.e., $\mathcal{A}(\omega, \delta H)=\mathcal{A}(\omega,-\delta H)$. The mode coupling at $\delta H=0$ therefore must generate a direct gap and parabolic dependence on small $\delta H$, as indicated in Fig. 4(b). Different radii break the symmetry and $\mathcal{A}(\omega, \delta H) \neq \mathcal{A}(\omega,-\delta H)$. Fig. 5(b) illustrates the strong magnon-magnon coupling of two different YIG spheres with $a_{1}=1 \mathrm{~mm}$ and $a_{2}=0.5 \mathrm{~mm}\left(\epsilon_{\mathrm{sp}} / \epsilon_{0}=15\right)$ in a cavity of radius $R=4 \mathrm{~mm}$. The asymmetry generates now an "indirect" gap. 
The radiative coupling transforms the individual magnon (Kittel) modes of the two-particle system into linear combinations, analogous to the molecular orbital theory of diatomic molecules, according to which the interaction of two atoms splits the levels into bonding (symmetric) and antibonding (antisymmetric) orbitals. The magnetic spheres can be interpreted as magnonic atoms that are bound into magnonic molecules. Particle arrays will form magnonic crystals, although this term is also used for magnetic structures with periodic variations of their magnetic properties [47, 48] or distributions of dipolarcoupled constituent materials [49]. The magnonic dimer has bonding and antibonding combinations, where the hybridization depends on the difference in their energies $\omega_{i}\left(H_{i}\right)$ and on their interaction. A homodimer $\mathrm{A}_{2}$ corresponds to Fig. 6(a), while the mismatched spheres in Fig. 6(b) form a heterodimer AB.

In a homodimer with inversion symmetry in which the splitting between internal modes is large, bonding is dominated by magnons with the same angular momentum $n$. We may use chemical intuition, however, to maximize the coupling by varying both the local field and the sphere radius. This may reduces the splitting between the internal $n=1$ and $n=2$ modes (cf. Fig. 7) and facilitate an increased bonding via sp-hybrid states.

Bonding and antibonding modes belong to different irreducible representations. In a heterodimer the lack of a mirror plane reduces the spatial symmetry and introduces couplings between all modes. Furthermore, energies of the different shells shift with respect to each other. Fig. 6 illustrates that the lowest-energy (dipolar) magnon of the smaller particle can couple efficiently to both the dipolar and higher multipolar magnons of the larger particle. The heterodimer thereby displays a significantly more complex magnon mixing behavior than the homodimer.

The bonding configuration corresponds to two dipole moments moving out of phase (optical mode, negative parity of dipole moments, or antisymmetric magnetic fields), while the antibonding configuration corresponds to the positive parity of the dipoles (acoustic mode, symmetric fields). In contrast to the positive parity (symmetric) magnons, the net magnetic moment of the negative parity (antisymmetric field) magnon vanishes for identical spheres, and does not interact with the $p$-wave cavity mode in the present configuration. The former are then bright, and the latter the dark states, as shown in Fig. (4). In the heterodimer, all magnons mix and contribute to the bonding and antibonding modes. As a consequence, all modes become bright, see Fig. (5).

We can parameterize the observations by elementary molecular orbital theory. The energy gap, $E_{\text {gap }}$, between the bonding and antibonding energy levels for a diatomic molecule is given by the secular equation

$$
E_{\text {gap }}^{2}=(2 g)^{2}+\left(E_{A}-E_{B}\right)^{2}
$$

where $g$ is the coupling parameter between the two sites, while $E_{A}$ and $E_{B}$ refer to their energies. There are two contributions to the energy gap, the covalent(homopolar)
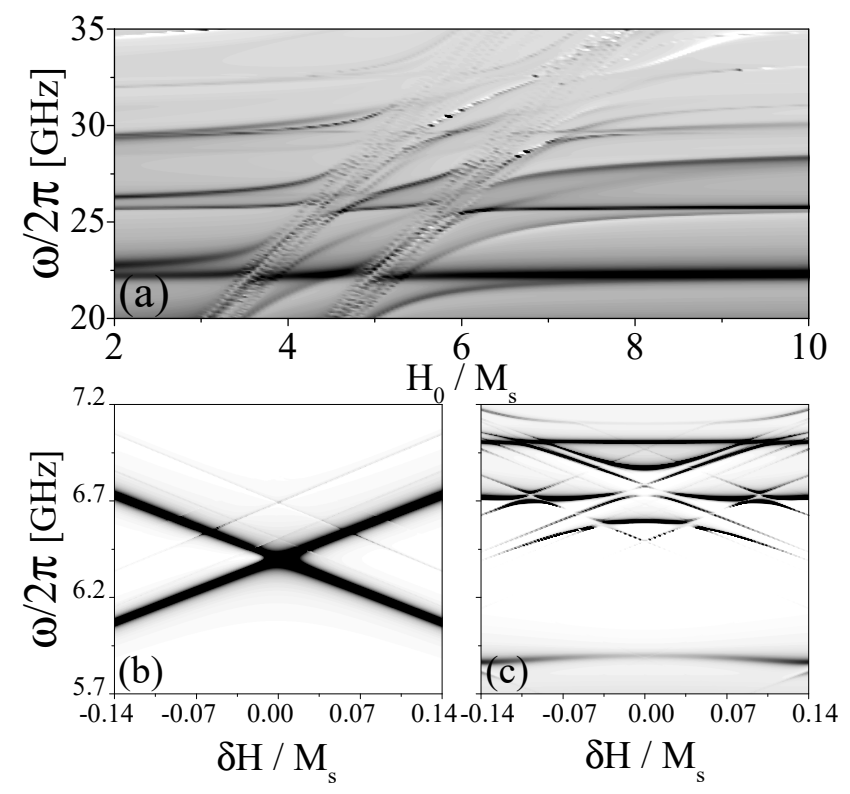

Figure 7. Same as Figs. 3 and 4 but for relatively large YIG spheres of radius $a_{1}=a_{2}=1.25 \mathrm{~mm}$. The cavity modes are strongly mixed with those confined in the two YIG spheres. In (a) the modes are shifted relative to each other by $\delta H / M_{s} \sim$ 0.7 and the uniform field in panels (b) and (c) is fixed at $H_{0} / M_{s} \sim 6$.

bonding contribution $E_{h}=2 g$, and the ionic contribution, $E_{i}=E_{A}-E_{B}$, due to the difference in "electronegativity" between the two atoms. For any bond, we can then define the bond covalency, $\alpha_{c}=E_{h} / E_{\text {gap }}$, and polarity, $\alpha_{p}=E_{i} / E_{\text {gap }}$, which parametrizes the continuous transition from covalent to ionic bonding.

In a homodimer at $\delta H=0$ we have a direct gap due to covalent bonding $E_{\text {gap }}=2 g$, see Fig. $4(\mathrm{~b})$ and bonding and anti-bonding wave functions are equally shared between the two atoms. However, in a heterodimer, due to the detuning of the atomic levels $\omega_{1}\left(H_{0}\right) \neq \omega_{2}\left(H_{0}\right)$, the gap has an "ionic" contribution, leading to an indirect gap as a function of $\delta H$ in Fig. 5(b). In a polar molecule, the amplitude of the bonding state shifts towards the more magnon-negative site referred to as the magnonic anion, with the anti-bonding state shifting towards the less magnon-negative site, referred to as the magnonic cation, a partially polarized molecule. The covalent bonding strength can be independently modulated by the average frequency spacing with the dominant cavity mode.

The scattering efficiency factor $Q_{\text {sca }}$ is plotted as a function of frequency $\omega / 2 \pi$, uniform field $H_{0} / M_{s}$, and differential field $\delta H / M_{s}$ for two YIG spheres of radius $a_{1}=a_{2}=1.25 \mathrm{~mm}$ and relative permittivity $\epsilon_{\mathrm{sp}} / \epsilon_{0}=15$, placed in a spherical cavity of radius $R=4 \mathrm{~mm}$ in Fig. 7 (a) and (c), and without cavity in $7(\mathrm{~b})$. Without cavity the system can be interpreted as two independent antennas operating in the ultrastrong coupling regime, since due to their relatively large size individual spheres act 
as efficient microwave antennas. Many anticrossings in Fig. 7(a) emphasize that the cavity modes are strongly and even ultrastrongly mixed with the modes in each individual spheres when detuned by a differential field $\delta H / M_{s} \sim 0.7$. The large differences between Figs. $7(\mathrm{~b})$ and (c) provide more evidence for the strong cavity-mode induced coupling between the spheres. In Fig. (7)(b), beside the main crossing modes in absence of the cavity, we observe tails from other crossings modes at higher frequencies, which are standing electromagnetic resonance modes confined by the magnetic spheres. Strong coupling with cavity mode not only turns the main crossing modes into anticrossing but also causes the complex anticrossing pattern shown in Fig. (7)(c) by hybridizing all higher modes.

\section{CONCLUSION}

In conclusion, we studied the plasmonics and optomagnonics of two dielectric and two magnetic spheres in microwave cavities by Mie scattering theory, i.e. a systematic expansion of the coupled Maxwell and LLG equations for magnetic systems. We employ the linear and magnetostatic approximations, but otherwise the treatment is numerically exact. The magnetization dynamics of spatially separated spheres in cavities can be efficiently coupled over large distances. The main reason is not the magnetic but the electric-field coupling, since two dielectric spheres with zero magnetization in a cavity display very similar dynamic behavior. Both strong and ultrastrong coupling can be realized not only for individual spheres but also for their mutual interaction. Two (properly placed) identical spheres form an inversion symmetric system, which is apparent by an anticrossing that generates a "direct" gap when plotted as a function of a symmetry breaking parameter, such as a staggered magnetic field or a size difference. Spheres with different sizes, however, break the symmetry at constant magnetic field and lead to an "indirect gap" as a function of field detuning. Magnon-polaritons within individual magnetic spheres may also hybridize in cavities, forming a complex mixed state of light and spin. Our study suggests a new direction for "spin cavitronics", viz. a route towards coherent control of the dynamics of various systems and materials (magnets, pieozoelectrics, superconductors, charge density waves, etc.) in microwave cavities via the nonmagnetic (plasmonic) interactions.

\section{ACKNOWLEDGMENTS}

B. Z. R. thanks S. M. Reza Taheri, A. Eskandari-asl, M. F. Miri and Y. M. Blanter for fruitful discussions. This work was partially supported by Iran Science Elites Federation (B. Z. R). Our research was supported by the Dutch NWO and JSPS Grants-in-Aid for Scientific Research (Grant Nos. 25247056, 25220910, 26103006).
[1] A. Wallraff, D. I. Schuster, A. Blais, L. Frunzio, R.-S. Huang, J. Majer, S. Kumar, S. M. Girvin, and R. J. Schoelkopf, Nature 431, 162 (2004).

[2] Y. Kubo, F. R. Ong, P. Bertet, D. Vion, V. Jacques, D. Zheng, A. Dréau, J. F. Roch, A. Auffeves, F. Jelezko, J. Wrachtrup, M. F. Barthe, P. Bergonzo, and D. Esteve, Phys. Rev. Lett. 105, 140502 (2010).

[3] S. Putz, D. O. Krimer, R. Amsüss, A. Valookaran, T. Nöbauer, J. Schmiedmayer, S. Rotter and J. Majer, Nature Physics 10, 720 (2014).

[4] N. Bar-Gill, L.M. Pham, A. Jarmola, D. Budker and R.L. Walsworth, Nature Communications 4, 1743 (2013).

[5] L. Childress, M. V. Gurudev Dutt, J. M. Taylor, A. S. Zibrov, F. Jelezko, J. Wrachtrup, P. R. Hemmer and M. D. Lukin, Science 314, 281 (2006).

[6] D. L. Mills and E. Burstein, Rep. Prog. Phys. 37817 (1974).

[7] A. Lehmeyer and L. Merten, J. Magn. Magn. Mater. 50, 32 (1985).

[8] Y. Cao, P. Yan, H. Huebl, S. T. B. Goennenwein, and G. E. W. Bauer, Phys. Rev. B 91, 094423 (2015).

[9] L. Bai, M. Harder, Y. P. Chen, X. Fan, J. Q. Xiao, and C.-M. Hu, Phys. Rev. Lett. 114, 227201 (2015).

[10] H. Maier-Flaig, M. Harder, R. Gross, H. Huebl, S. T. B. Goennenwein, Phys. Rev. B 94, 054433 (2016)

[11] B. Zare Rameshti, Y. Cao, and G. E. W. Bauer, Phys. Rev. B 91, 214430 (2015).
[12] Y. Tabuchi, S. Ishino, T. Ishikawa, R. Yamazaki, K. Usami, and Y. Nakamura, Phys. Rev. Lett. 113, 083603 (2014); Y. Tabuchi, S. Ishino, A. Noguchi, T. Ishikawa, R. Yamazaki, K. Usami, and Y. Nakamura, Science 349, 6246 (2015).

[13] X. Zhang, C.-L. Zou, L. Jiang, and H.X. Tang, Phys. Rev. Lett. 113, 156401 (2014).

[14] T. Niemczyk et al., Nature Physics 6, 772 (2010).

[15] Y. R. Shen and N. Bloembergen, Phys. Rev. 143, 372 (1966).

[16] S.O. Demokritov, B. Hillebrands, A. N. Slavin, Physics Reports 348, 441-489 (2001).

[17] X. Zhang, N. Zhu, C.-L. Zou, and H. X. Tang, Phys. Rev. Lett. 117, 123605 (2016).

[18] A. Osada, R. Hisatomi, A. Noguchi, Y. Tabuchi, R. Yamazaki, K. Usami, M. Sadgrove, R. Yalla, M. Nomura, and Y. Nakamura, Phys. Rev. Lett. 116, 223601 (2016).

[19] M. A. Ruderman and C. Kittel, Phys. Rev. 96 , 99 (1954).

[20] T. Kasuya, Prog. Theor. Phys. 16, 45 (1956).

[21] K. Yosida, Phys. Rev. 106, 893 (1957).

[22] S. S. P. Parkin, N. More, and K. P. Roche, Phys. Rev. Lett. 64, 2304 (1990).

[23] P. Bruno and C. Chappert, Phys. Rev. Lett. 67, 1602 (1991).

[24] S. S. P. Parkin and D. Mauri, Phys. Rev. B 44, 7131 (1991).

[25] S. Haroche and J.-M. Raimond, Exploring the Quantum: Atoms, Cavities and Photons (Oxford University Press, 
Oxford, 2006).

[26] A. Blais, R.-S. Huang, A. Wallraff, S. M. Girvin, and R. J. Schoelkopf, Phys. Rev. A 69, 062320 (2004).

[27] M. Gilleo and S. Geller, Phys. Rev. 110, 73 (1958).

[28] Y. Kajiwara, K. Harii, S. Takahashi, J. Ohe, K. Uchida, M. Mizuguchi, H. Umezawa, H. Kawai, K. Ando, K. Takanashi, S. Maekawa, and E. Saitoh, Nature 464, 262 (2010).

[29] B. Heinrich, C. Burrowes, E. Montoya, B. Kardasz, E. Girt, Y.-Y. Song, Y. Sun, and M. Wu, Phys. Rev. Lett. 107, 066604 (2011).

[30] H. Kurebayashi, O. Dzyapko, V.E. Demidov, D. Fang, A.J. Ferguson, and S.O. Demokritov, Nature Materials 10, 660 (2011).

[31] H. Huebl, C.W. Zollitsch, J. Lotze, F. Hocke, M. Greifenstein, A. Marx, R. Gross, and S.T.B. Goennenwein, Phys. Rev. Lett. 111, 127003 (2013).

[32] G.B.G. Stenning, G.J. Bowden, L.C. Maple, S. A. Gregory, A. Sposito, R.W. Eason, N.I. Zheludev, and P.A.J. de Groot, Opt. Exp. 21, 1456 (2013).

[33] B. Bhoi, T. Cliff, I.S. Maksymov, M. Kostylev, R. Aiyar, N. Venkataramani, S. Prasad, and R.L. Stamps, J. Appl. Phys. 116, 243906 (2014).

[34] M. Goryachev, W.G. Farr, D.L. Creedon, Y. Fan, M. Kostylev, and M.E. Tobar, Phys. Rev. Applied, 2, 054002 (2014).

[35] Ö. O. Soykal and M. E. Flatté, Phys. Rev. Lett. 104, 077202 (2010); Phys. Rev. B 82, 104413 (2010).

[36] J. Bourhill, N. Kostylev, M. Goryachev, D. L. Creedon, and M. E. Tobar, Phys. Rev. B 93, 144420 (2016).
[37] N. J. Lambert, J. A. Haigh, S. Langenfeld, A. C. Doherty, and A. J. Ferguson, Phys. Rev. A 93, 021803(R) (2016).

[38] L. Bai, M. Harder, P. Hyde, Z. Zhang, C.-M. Hu, Y. P. Chen, and J. Q. Xiao, Phys. Rev. Lett. 118, 217201 (2017).

[39] L. R. Walker, J. Appl. Phys. 29, 318 (1958).

[40] P.C. Fletcher and R.O. Bell, J. Appl. Phys., 30, 687 (1959).

[41] G. Mie, Ann. Phys., 330: 377 E45 (1908).

[42] J. A. Stratton, "Electromagnetic Theory" Wiley-IEEE Press (2007).

[43] Yu-lin Xu, Journal of Computational Physics 127, 285 298 (1996).

[44] S. A. Manuilov, S. I. Khartsev, and A. M. Grishin, J. Appl. Phys. 106, 123917 (2009).

[45] K. Sadhana, R. S. Shinde, and S. R. Murthy, Int. J. Mod. Phys. B 23, 3637 (2009).

[46] E. Prodan, C. Radloff, N. J. Halas, and P. Nordlander, Science 302, 419 (2003).

[47] M. Krawczyk and D. Grundler, J. Phys.: Condens. Matter 26, 123202 (2014).

[48] A. V. Chumak, T. Neumann, A. A. Serga, B. Hillebrands and M. P. Kostylev, J. Phys. D: Appl. Phys. 42, 205005 (2009).

[49] J. O. Vasseur, L. Dobrzynski, B. Djafari-Rouhani, and H. Puszkarski, Phys. Rev. B 54, 1043 (1996). 